\title{
Shaping light beams in the nanometer scale: A Yagi-Uda nanoantenna in the optical domain
}

\author{
Jingjing Li, Alessandro Salandrino, and Nader Engheta* \\ Department of Electrical and Systems Engineering, University of Pennsylvania, Philadelphia, Pennsylvania 19104, USA
}

(Received 26 January 2007; revised manuscript received 7 June 2007; published 6 December 2007)

\begin{abstract}
A Yagi-Uda-like optical nanoantenna concept using resonant core-shell plasmonic particles as its "reflectors" and "directors" is studied numerically. Such particles when placed near an optical dipole source in a certain arrangement may exhibit large induced dipole moments, resulting in shaping the far-field radiation pattern, analogous to the far field of classical Yagi-Uda antennas in the microwave regime. The variation of the ratio of radii in concentric core-shell nanostructure is used to tailor the phase of the polarizabilities of the particles and, consequently, the antenna's far-field pattern. The idea of a nanospectrum analyzer is also briefly proposed for molecular spectroscopy.
\end{abstract}

DOI: 10.1103/PhysRevB.76.245403

PACS number(s): 73.20.Mf, 07.60.Rd, 78.67.Bf, 84.40.Ba

\section{INTRODUCTION}

Plasmonic materials have generated increasing interest in various research communities in recent years due to their ability to manipulate optical signals at deep subwavelength dimensions (for a review, see Ref. 1). One of their interesting features is related to the scattering properties of subwavelength plasmonic nanoparticles. It is well known that for a particle made of a plasmonic material, the scattering of electromagnetic waves is maximized at a certain resonant wavelength determined by the material parameters and the particle geometry, even though the size of the particle may be much smaller than the free-space wavelength. The peculiar characteristics of interaction of light with plasmonic nanoparticles have been known for a long time, and recently, owing to the advancement in nanofabrication technologies, the interest in the scattering resonance associated with the presence of plasmonic nanoparticles has been resurrected and explored in detail, both experimentally and theoretically. ${ }^{2-5}$ The influence of the plasmonic nanoparticles on the single-molecule spontaneous emission has also been studied, where the stimulated molecule was modeled as a dipole source (see Refs. 6 and 7, and references therein). These studies have been mostly concentrated on the emission rate of the molecule (i.e., dipole) in a certain environment and only a subset of them is concerned with the modification to the radiation pattern (e.g., Refs. 8 and 9).

Design and fabrication of optical nanoantennas have gained growing interest in the recent years. ${ }^{10-12}$ It is a challenging task, mainly due to the optical properties of metals that are significantly different from their microwave characteristics. Instead of exploiting their high-conductivity property as in the radio frequency (rf) and microwave domains, their plasmonic features in the optical regime became one of the relevant factors in nanoscale optical antenna design. Motivated and inspired by the various concepts of antenna theory in rf and microwave regimes, such as the conventional rf Yagi-Uda antennas, in a recent work, we suggested that plasmonic resonance of core-shell plasmonic particles can be exploited for optical antenna array design. ${ }^{13}$ In this idea, a group of resonant plasmonic core-shell nanoparticles is placed around an optical dipole source $p_{0}$ in a specified pattern. Strong dipole moments may be induced on these particles such that the overall far-field radiation characteristics may become different from that of a single dipole source, analogous to what occurs in a conventional microwave Yagi-Uda antenna. The optical dipole source can be a stimulated molecule, a quantum dot, or the tip of a near-field scanning optical microscope. In the present work, we explore this concept in detail using analytical technique and numerical simulations. ${ }^{20}$

\section{PLASMONIC NANOSHELL PARTICLES AT SCATTERING RESONANCE AS OPTICAL ANTENNA ELEMENTS}

The scattering resonance of a concentric plasmonic coreshell spherical nanoparticle has been extensively studied experimentally ${ }^{3}$ and theoretically. ${ }^{5}$ When the size of this particle is much smaller than the incident wavelength, its interaction with the electromagnetic wave is modeled by an induced electric dipole moment with the polarizability $\alpha$ given as $\alpha=-6 \pi i \epsilon_{0} c_{1}^{\mathrm{TM}} / k_{0}^{3}$, where $k_{0}$ is the free-space wave number (or the wave number in the surrounding medium) and $c_{1}^{\mathrm{TM}}$ the scattering coefficient of the TM mode of order 1 in the Mie scattering analysis (see Ref. 5 for more details), whose value is given as $c_{n}^{\mathrm{TM}}=-U_{n}^{\mathrm{TM}} /\left(U_{n}^{\mathrm{TM}}+i V_{n}^{\mathrm{TM}}\right)$ with $n$ $=1$, where $U_{n}^{\mathrm{TM}}$ is

$$
U_{n}^{\mathrm{TM}}=\left|\begin{array}{cccc}
j_{n}\left(k_{1} b\right) & j_{n}\left(k_{2} b\right) & y_{n}\left(k_{2} b\right) & 0 \\
\tilde{j}_{n}\left(k_{1} b\right) / \epsilon_{1} & \tilde{j}_{n}\left(k_{2} b\right) / \epsilon_{2} & \tilde{y}_{n}\left(k_{2} b\right) / \epsilon_{2} & 0 \\
0 & j_{n}\left(k_{2} a\right) & y_{n}\left(k_{2} a\right) & j_{n}\left(k_{0} a\right) \\
0 & \tilde{j}_{n}\left(k_{2} a\right) / \epsilon_{2} & \tilde{y}_{n}\left(k_{2} a\right) / \epsilon_{2} & \tilde{j}_{n}\left(k_{0} a\right) / \epsilon_{0}
\end{array}\right|
$$

and $e^{-i \omega t}$ convention is assumed. Here, $j_{n}(x)$ and $y_{n}(x)$ are the $n$th order spherical Bessel functions of the first and second kind. $\tilde{j}_{n}(x)$ stands for $\partial\left[x j_{n}(x)\right] / \partial x$, and $\tilde{y}_{n}(x)$ is similarly defined. $\epsilon_{1}$ and $\epsilon_{2}$ are the permittivity of the core and of the shell, respectively, while $k_{1}$ and $k_{2}$ are the wave numbers in each respective region. The outer and inner radii of the particle are denoted as $a$ and $b . V_{n}^{\mathrm{TM}}$ is similar to $U_{n}^{\mathrm{TM}}$, except the functions $\tilde{j}_{n}$ and $j_{n}$ in the last column are replaced by $\tilde{y}_{n}(x)$ and $y_{n}$, respectively. The maximum of the magnitude of 


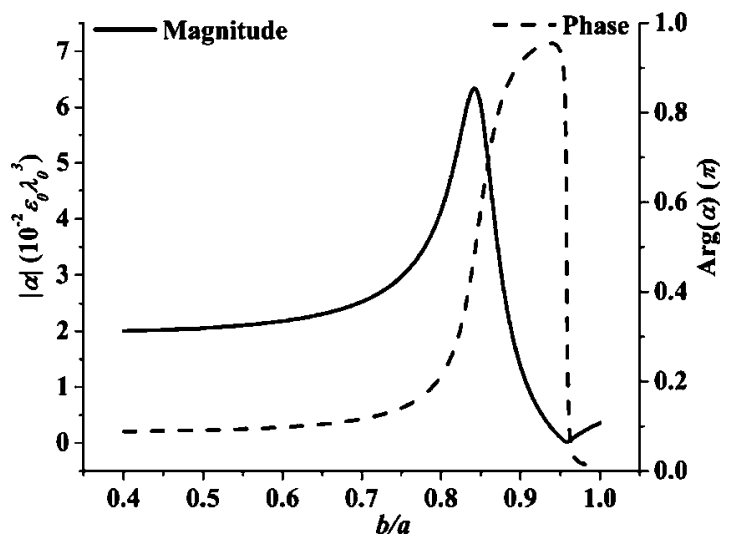

FIG. 1. Magnitude and phase of the polarizability $\alpha$ of a concentric nanoshell particle vs $b / a$. Operating wavelength is $620 \mathrm{~nm}$. The core is made of $\mathrm{SiO}_{2}\left(\epsilon_{1}=2.2 \epsilon_{0}\right)$ and the shell of silver $\left[\epsilon_{2}=(-15.33+0.451 i) \epsilon_{0}\right]$.

$\alpha$ is reached when $V_{1}^{\mathrm{TM}}$ attains a zero value. A necessary condition for $V_{1}^{\mathrm{TM}}=0$ is to have $\epsilon_{1}$ and $\epsilon_{2}$ of real values with opposite signs. At a given wavelength for which the imaginary part of the permittivity of the plasmonic material is small compared to the real part, the condition $V_{1}^{\mathrm{TM}}=0$ can be achieved approximately by adjusting $b / a$ (see Fig. 1).

It is well known that for a subwavelength uniform sphere made of a given plasmonic material, local plasmonic resonance is achieved when the real part of the relative permittivity (with respect to that of the surrounding medium) is close to -2 . However, by adjusting the ratio $b / a$, the scattering resonance can be tailored at other wavelength range for a concentric nanoparticle. When such particles, which are placed around an optical dipole source $p_{0}$, are designed to resonate at the dipole's operating wavelength, the local field inducing the dipole moment on each particle is the superposition of the fields emitting from the original dipole source $p_{0}$ and all other induced dipoles. Thus, the induced dipole $p_{i}$ on the $i$ th particle can be written as

$$
\boldsymbol{p}_{i}=\alpha_{i}\left(\sum_{j \neq i} \underset{\underline{\Gamma}}{\underline{\underline{n}}}\left(\boldsymbol{r}_{i}, \boldsymbol{r}_{j}\right) \cdot \boldsymbol{p}_{j}+\underline{\underline{\Gamma}}\left(\boldsymbol{r}_{i}, \mathbf{0}\right) \cdot \boldsymbol{p}_{0}\right),
$$

where $\boldsymbol{r}_{i}$ and $\boldsymbol{r}_{j}$ are the position vectors of particles $i$ and $j$, respectively. $\underline{\underline{\Gamma}}\left(\boldsymbol{r}_{i}, \boldsymbol{r}_{j}\right)$ is the dyadic Green function of the dipole $\boldsymbol{p}_{j}$ evaluated at the position of the $i$ th particle, which is known when the positions of all the particles and the source dipole are given. The original optical dipole source is expressed by $\boldsymbol{p}_{0}$, and its field on dipole $i$ is written separately as the second term in the right hand side. This system of linear equations can be solved directly. We would like to emphasize that Eq. (2) takes into account the retardation effects, and no quasistatic approximation is assumed here. The far-field radiation pattern of the system, thus, is equivalent to that of the $N+1$ dipole system - the source dipole and $N$ induced dipoles - with the magnitude and phase achieved from Eq. (2). An example is shown in Fig. 2. For this system, two identical concentric plasmonic particles are placed at each side of the optical dipole $p_{0}$ (whose vector is in the $\hat{z}$ direction) at a distance of $0.35 \lambda_{0}$, with a free-space wavelength
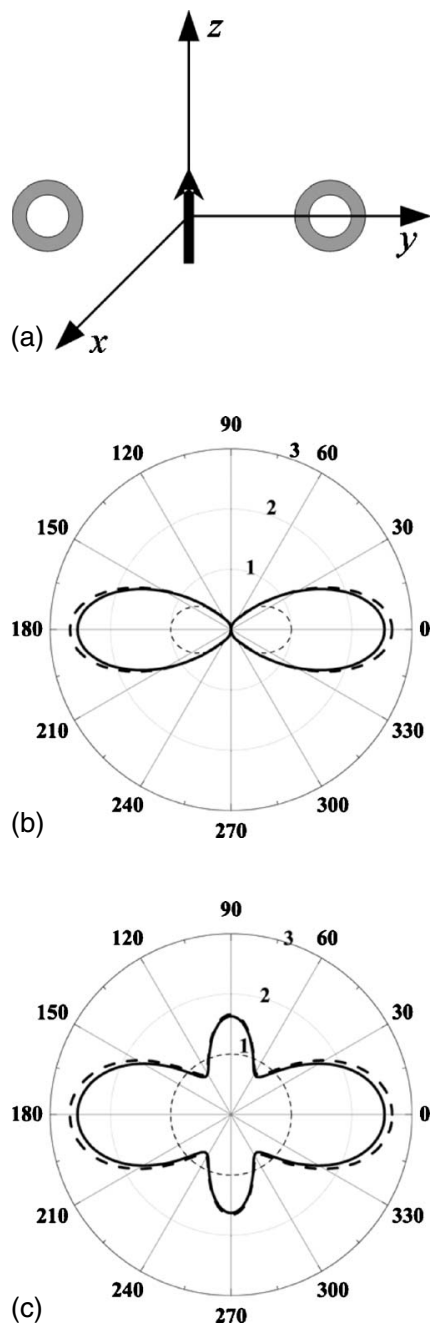

FIG. 2. An optical antenna array composed of two identical plasmonic core-shell nanoparticles placed on both sides of an optical dipole source. (a) The geometry of the problem. Pattern of the radiated power flux density (b) in the $E$ plane and (c) in the $\mathrm{H}$ plane. Bold solid lines represent the radiation pattern of the system using dipole approximation. Bold dashed lines are for the case when the influence of electric quadrupole and magnetic dipole is also included. Light dashed lines are the radiation patterns of the source dipole alone. The patterns are normalized with respect to the maximum of the radiated power flux density of the original dipole source when it radiates in the absence of the particles.

$\lambda_{0}=620 \mathrm{~nm}$ [Fig. 2(a)]. The cores of the particles are assumed to be made of $\mathrm{SiO}_{2}$ with $\epsilon_{1}=2.2 \epsilon_{0}$, the shells are made of silver with $\epsilon_{2}=(-15.33+0.451 i) \epsilon_{0}$ at this operating wavelength, ${ }^{14} a=0.1 \lambda_{0}$, and $b / a=0.842$. For these parameters, the polarizability is $\alpha=0.063 e^{i 0.48 \pi} \epsilon_{0} \lambda_{0}^{3}$. The pattern of radiated power is given in Figs. 2(b) and 2(c) which is normalized to the maximum point of radiated power flux density of the original source dipole when it radiates in the absence of particles. It can be clearly seen that, as expected, both the magnitude and the shape of the pattern are modified by the presence of the particles when compared with the pattern of the original dipole source alone. In this sense, the plasmonic particles take the role of an antenna array at optical wavelengths. 
In the above calculations, the particles have been modeled as induced electric dipoles. A more accurate description of the system should take into account the higher-order multipoles on each particle. We give the mathematical details of this approach in the Appendix. The radiated power pattern of the system when the electric quadrupole and magnetic dipole are also included is shown in Figs. 2(a) and 2(c) as bold dashed lines. Since the dipolar scattering coefficient is ordinarily much higher than the scattering coefficients of all other higher-order modes when the particles are excited around dipolar scattering resonance, the influence of the higher-order multipolar scattering is indeed relatively very small as long as the particles are not very close to the dipole source or to each other, as we clearly see in the power pattern plots in Figs. 2(a) and 2(c). This justifies our design approach of the optical antenna, which is based on the induced dipole approximation of the plasmonic particles. In the rest of this paper, we will continue using this dipole approximation for the antenna design, and we use the higher-order approximation (with electric quadrupole and magnetic dipole included) to test the adequacy of the model.

\section{OPTICAL YAGI-UDA ANTENNAS COMPOSED OF PLASMONIC PARTICLES}

In the field of antenna design, it is commonly required to design an antenna system with a prescribed far-field radiation pattern. For optical nanoantennas using plasmonic particles as described above, the induced dipole moment on each particle is resulted from the fields of the source and all the other induced dipoles. This implies that all the induced dipoles are essentially coupled to one another such that we do not have an arbitrarily separate control over the phase and magnitude of each dipole element individually. This makes the pattern synthesis problem for the collection of plasmonic particles in the optical antenna array a challenging, but still achievable task. An interesting analogous case of such a pattern synthesis in the microwave domain is the celebrated Yagi-Uda antenna. ${ }^{15,16}$ In this design, as shown in Fig. 4(a), a halfwavelength resonant dipole antenna is driven by a source (e.g., a voltage source), and several other wire elements with proper lengths, performing as passive elements, are placed at specific positions in proximity of the driven dipole in order to reshape the far-field radiation pattern. One of these passive wire elements, longer than the resonating length, is placed at one side of the source antenna. This element with an inductive impedance response works as a "reflector." Several other wire elements with lengths shorter than the resonating length are situated at the other side of the driven source. These shorter elements, which are called directors, have capacitive impedance response. The resulting radiation pattern of such an antenna has a narrow beam toward the direction of the "directors" and a minimum (or a null) toward the reflector direction. The essential idea in the classic Yagi-Uda antenna was to introduce some break in the symmetry of the response phases of the scatterers placed at each side of the source so that the shape of the total radiation distribution exhibits a certain break in the symmetry and more directivity. Such an idea may be transplanted into our optical nanoantenna array design, where the core-shell nanoparticles can play the roles of reflectors and directors. Ordinarily, the values of ratio of the radii $b / a$ for the core-shell nanoparticle are designed to set the particles at scattering resonance. Under this condition, the phase of the polarizability is close to $\pi / 2$, and the equivalent current of the induced dipole is almost in phase with the incident field. However, $b / a$ can be designed deliberately to "detune" this resonance in order to achieve a phase of induced dipole less than or greater than $\pi / 2$ compared to the incident field. Therefore, these detuned nanoparticles may play the role of the reflector or the director element in the Yagi-Uda antenna. By placing one reflector nanoparticle at one side of the original dipole source, and one or several director nanoparticles at the opposite side, we can design a Yagi-Uda antenna at optical wavelengths.

To test our idea, we first show a design of two-particle Yagi-Uda antenna working at $620 \mathrm{~nm}$. Both particles have the core of $\mathrm{SiO}_{2}$ and the shell of silver as those in Fig. 2. The outer radius of each particle is still $0.1 \lambda_{0}$. However, this time, for the particle at the left, we use $b / a=0.851$ so that $\alpha_{1}=0.0592 e^{i 0.6 \pi} \epsilon_{0} \lambda_{0}^{3}$, while for the one at the right, we use $b / a=0.834$ so that $\alpha_{2}=0.0611 e^{i 0.4 \pi} \epsilon_{0} \lambda_{0}^{3}$. Notice that the phases of the polarizabilities are shifted to be above or below $\pi / 2$. The two particles are placed at equal distance $d$ from the source dipole. Then $d$ is varied to achieve a "proper" radiation pattern, which should have a small value at $0^{\circ}$ or $180^{\circ}$, and a large value at the opposite. The pattern of one of these designs is shown as the bold solid line in Fig. 3, for which $d=0.25 \lambda_{0}$. We declare the particle at the left side an "inductive" element because the effective induced current of the dipole has a phase that leads with respect to the phase of the incident field. For a similar reason, the particle at the right is a "capacitive" one. For this case, we obtain a radiation pattern which is maximized at the $0^{\circ}$ direction and is smaller at the opposite direction, which is the inductive particle side. The inductive particle at the left essentially works as the reflector, while the capacitive particle on the right works as the director. The conceptual idea is analogous to the conventional Yagi-Uda antenna; that is, by introducing some asymmetry in the two elements placed at the opposite sides of the source dipole, it may be possible to shape the beam patterns accordingly. The radiated power patterns when higher-order multipoles (up to electric quadrupole and magnetic dipole) are included are also shown as the bold dashed line. Again, we notice that, in this example, these patterns are very close to those of the dipole approximation.

Yagi-Uda optical antennas with more than one director can also be designed in order to achieve a narrower beam pattern. In this case, several degrees of freedom are involved and we need to optimize the antenna design heuristically. Suppose we want to design a one-reflector, eight-director Yagi-Uda optical antenna. One of the design processes begins from the two-particle Yagi-Uda optical antenna shown in Fig. 3, with the particle-to-dipole-source distance as $d$ $=0.25 \lambda_{0}$, for which the $0.6 \pi$-phase particle works as a reflector and the $0.4 \pi$-phase particle works as a director. To design a multiple-director Yagi-Uda optical antenna, we use the same reflector and fix the reflector-source distance to be $d_{1}$ $=0.25 \lambda_{0}$. Suppose the distances between each directors are the same, and is equal to the distance between the dipole 


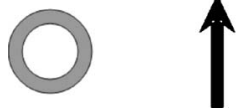

(a)

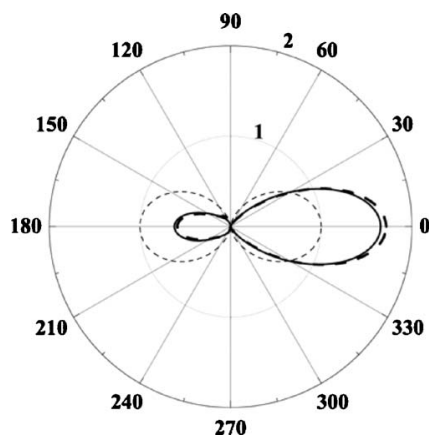

(b)

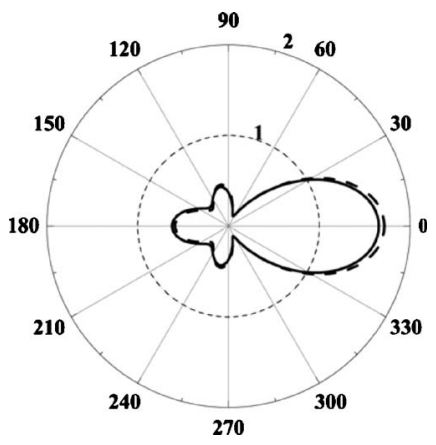

(c)

FIG. 3. A two-particle optical Yagi-Uda nanoantenna operating at $620 \mathrm{~nm}$. (a) The geometry. Pattern of the radiated power flux density (b) in the $E$ plane and (c) in the $H$ plane. Bold solid lines show the pattern of the system using dipole approximation. Bold dashed lines represent the pattern when the influence of electric quadrupole and magnetic dipole are also included. The light dashed line is for the source dipole alone. The patterns are normalized as described in the caption of Fig. 2.

source and the first director $d_{2} . d_{2}$ is then varied to achieve a proper pattern. The pattern of a "good" design for $d_{2}$ $=0.65 \lambda_{0}$ is shown in Fig. 4 as the bold solid line. Clearly, a narrower beam is achieved compared to that in Fig. 3. The patterns for which the influence of electric quadrupole and magnetic dipole is also included are shown as bold dashed lines, highlighting the fact that the dipole approximation is adequate for this example.

The patterns of this nanoantenna system at different operating wavelengths are shown in Fig. 5. In this analysis, for the dielectric function of the silver part of the nanoshell particles, we consider a Drude model that is fitted to the available experimental data for silver permittivity. ${ }^{14}$ We noticed that such a system yields an even narrower beam at a slightly different operating wavelength $\lambda_{0}=646 \mathrm{~nm}$, as shown in Fig. 5(c). Such a pattern is much more attractive than that of Fig. 2 and 3 when a higher directivity is desired. The patterns possess similar forms in a certain range of operating wavelengths [see Figs. 4(b) and 4(c) for the $620 \mathrm{~nm}$ pattern and Figs. 5(b) and 5(c) for the 674 and $646 \mathrm{~nm}$ patterns]. As we
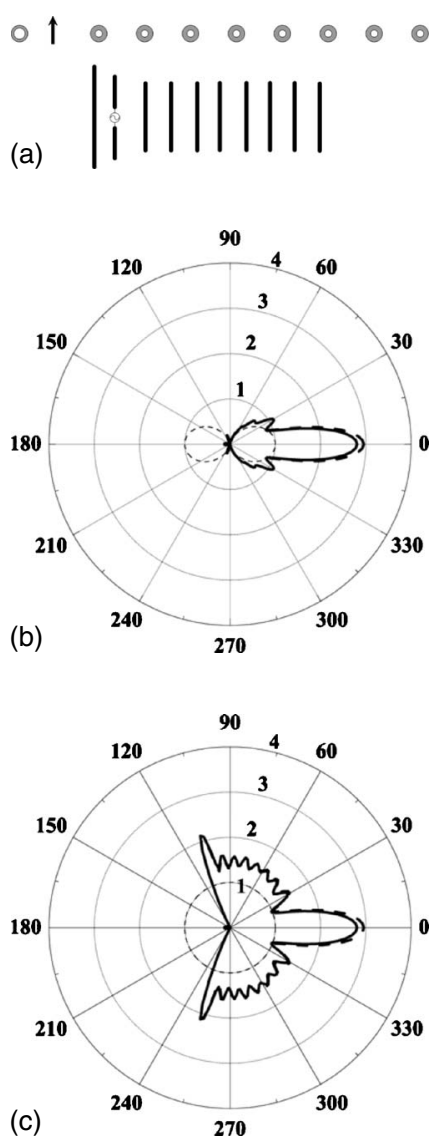

FIG. 4. A nine-particle optical Yagi-Uda nanoantenna. (a) The geometry of the optical Yagi-Uda antenna and the diagram of a classical Yagi-Uda antenna in the rf and microwave domains. Pattern of the radiated power flux density (b) in the $E$ plane and (c) in the $H$ plane. Bold solid line, the pattern using dipole approximation; bold dash line, the pattern when the influence of electric quadrupole and magnetic dipole is included; and light dashed line, the pattern of the dipole source alone.

shift away from the design wavelength, such as at $750 \mathrm{~nm}$ [Fig. 5(a)] or $500 \mathrm{~nm}$ [Fig. 5(d)], the pattern obviously loses its directivity, and its magnitude is also much smaller. A detailed study on the influence of design parameters, such as the ratio of radii, the position of the reflector and directors, etc., is indeed of great interest, but is out of the scope of this paper.

\section{DISCUSSION}

From a practical point of view, one may be concerned about how to precisely fabricate the core-shell structure with different shell thicknesses for the reflector or the director(s). It seems a challenging task for the present technology to control the thickness of the shell at a precision below $1 \mathrm{~nm}$ as may be required in our theoretical analysis, although the technology of nanoshell synthesis with controllable geometry parameters is developing fast. However, if the ratio of radii of the fabricated concentric particles has a wide distribution around the desired values in a real experimental condition, one can, in principle, use the scattering resonance 

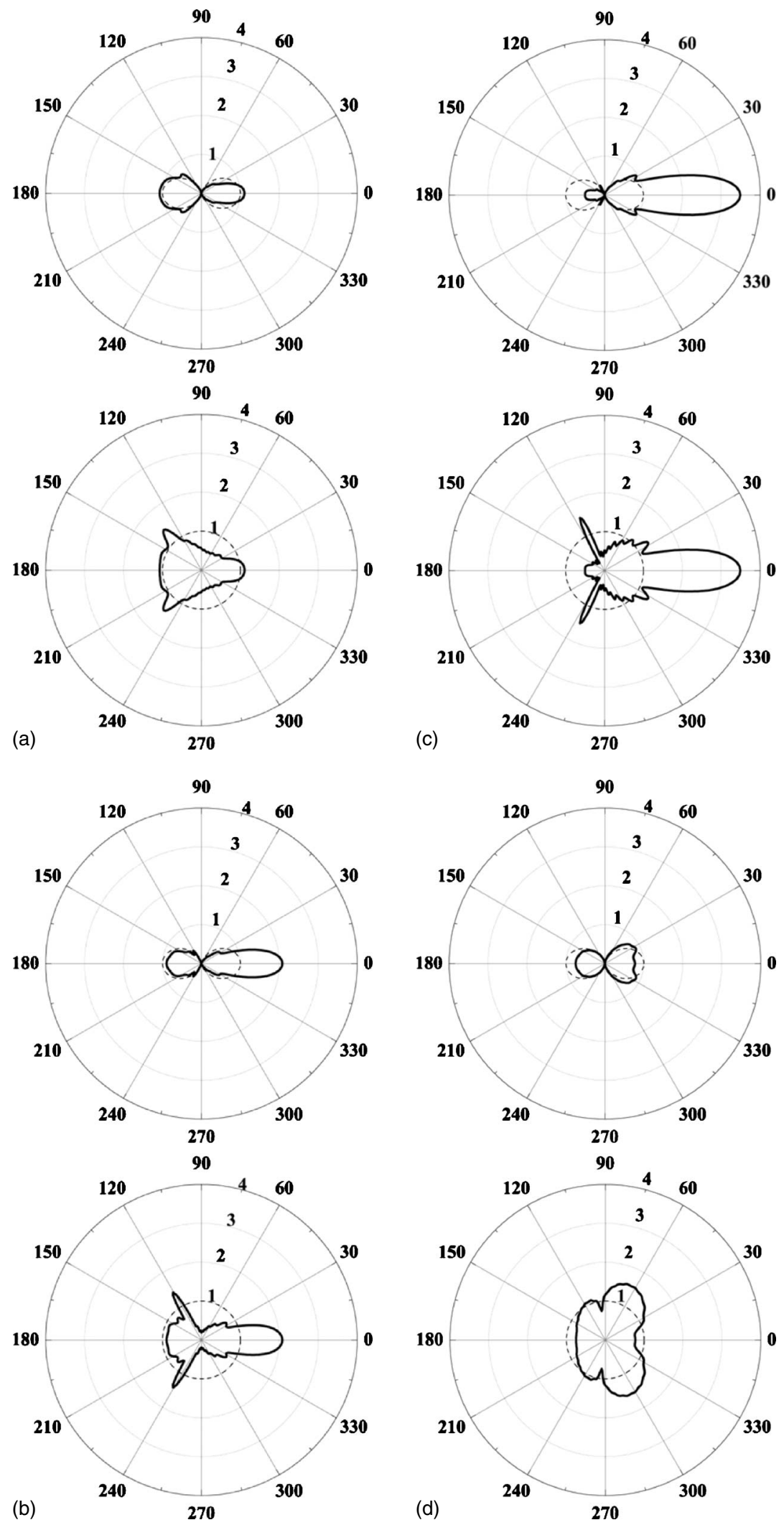

FIG. 5. Pattern of the radiated power flux density of the Yagi-Uda antenna shown in Fig. 4(a) at different operating wavelengths: (a) $750 \mathrm{~nm}$, (b) $674 \mathrm{~nm}$, (c) $646 \mathrm{~nm}$, and (d) $500 \mathrm{~nm}$. The upper row is the $E$ plane, while the lower row is the $H$ plane. Solid lines, pattern of the Yagi-Uda antenna; dashed lines, pattern of a single dipole. 


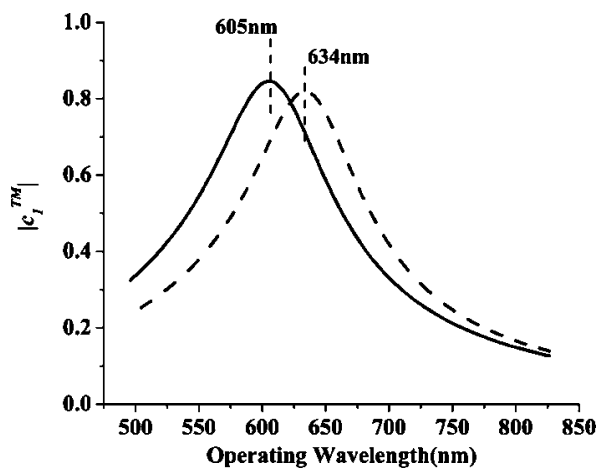

FIG. 6. The dispersion property of $c_{1}^{\mathrm{TM}}$ for a single concentric particle of outer radius $62 \mathrm{~nm}$, with core made of $\mathrm{SiO}_{2}$ and shell made of silver. The radii ratio is 0.851 for the dashed line, and 0.834 for the solid line. Silver follows a Drude model in this calculation.

wavelength of each particle as the criterion to identify and select the proper particles for the reflector or the director(s) of interest. In fact, the reflector and the director have different scattering spectra (Fig. 6), and with the outer diameter we use in the former description $(124 \mathrm{~nm})$, a particle with core/ shell radii ratio of 0.851 gives a scattering peak at $634 \mathrm{~nm}$, longer than the operating wavelength of $620 \mathrm{~nm}$, while the particle with 0.834 radii ratio resonate at $605 \mathrm{~nm}$, shorter than the operating wavelength. By testing the particles one by one using modern near-field technology, ${ }^{17-19}$ one can, in principle, find the desired reflector and directors. We also point out that although the scattering property of the concentric particles may change rapidly with the ratio of radii due to their small size, the radiated power pattern of the entire array may maintain its general shape over a certain range of operating wavelengths $(620-674 \mathrm{~nm})$. This is due to the fact that the scattering strength of the concentric nanoparticles may vary less rapidly with wavelength, as shown in Fig. 6. It must be pointed out that the pattern of our array system analyzed in Fig. 4 is reasonably robust to a limited uncertainty in the particles' position as long as the deviation from the ideal positions is within a few percent of the particle's size. (Results are not shown here for the sake of brevity.)

As the operating wavelength shifts out of the operating band, the radiation intensity decreases, and the pattern will no longer have the dominant beam along $180^{\circ}$. This is because not only are the polarizabilities of each particle frequency dependent, but also, at different wavelengths, the relative distances between the source dipole and the particles are electrically different (while they are physically fixed), and thus, the coupling among the particles is changed with wavelength. Such a wavelength dependence of the beam patterns can offer exciting potential applications in molecular fluorescence. The emission spectrum of a stimulated molecule may often cover a wide wavelength range, thus a stimulated molecule may emit as a broadband source. One can design a set of more than one Yagi-Uda nanoantenna array driven by the same molecular source, but each antenna optimized at different wavelengths such that the maximum beam at a given wavelength points to a different direction. This can essentially lead to the analysis of the spectral infor- mation of the molecular emission, transforming the spectral contents into angular variation of emitted signals, and thus, effectively providing a "nano spectrum analyzer" at the nanometer scale at optical wavelengths. The details of our analysis for such a device, which can have various potential applications in molecular spectroscopy and sensing, will be given in a future publication.

\section{ACKNOWLEDGMENT}

This work was supported in part by the U.S. Air Force Office of Scientific Research (AFOSR) Grant No. FA955005-1-0442.

\section{APPENDIX: HIGH-ORDER MULTIPOLES INDUCED ON THE PARTICLE}

Suppose the source dipole is aligned along the $\hat{z}$ direction. With the configuration of Fig. 2(a), we can easily notice that on each particle the induced electric dipole has only a $\hat{z}$ component, the induced electric quadrupole has only a $\hat{y} \hat{z}$ component, and the induced magnetic dipole has only an $\hat{x}$ component. We use $p_{i}, q_{i}$, and $m_{i}$ (all are scalars) to indicate the magnitude of the induced electric dipole, induced electric quadrupole, and induced magnetic dipole on particle $i$. We can then write the function of the system in scalar form including the influence of the induced electric dipole, electric quadrupole, and magnetic dipole as

$$
\begin{gathered}
p_{i}=\alpha_{p i} \sum_{j \neq i}\left[F_{p p}\left(\boldsymbol{r}_{i j}\right) p_{j}+F_{p q}\left(\boldsymbol{r}_{i j}\right) q_{j}+F_{p m}\left(\boldsymbol{r}_{i j}\right) m_{j}\right], \\
q_{i}=\alpha_{q i} \sum_{j \neq i}\left[F_{q p}\left(\boldsymbol{r}_{i j}\right) p_{j}+F_{q q}\left(\boldsymbol{r}_{i j}\right) q_{j}+F_{q m}\left(\boldsymbol{r}_{i j}\right) m_{j}\right], \\
m_{i}=\alpha_{m i} \sum_{j \neq i}\left[F_{m p}\left(\boldsymbol{r}_{i j}\right) p_{j}+F_{m q}\left(\boldsymbol{r}_{i j}\right) q_{j}+F_{m m}\left(\boldsymbol{r}_{i j}\right) m_{j}\right] .
\end{gathered}
$$

In this equation, $F_{p q}\left(\boldsymbol{r}_{i j}\right)$ is the scalar interacting function from quadrupole $q_{j}$ to dipole $p_{i}$, and the other $F$ 's in front of $p_{j}, q_{j}$, and $m_{j}$ have similar meanings. Since $p_{i}$ is proportional to $E_{z}, q_{i}$ is proportional to $\partial_{y} E_{z}+\partial_{z} E_{y}$, and $m_{i}$ is proportional to $H_{x}$ (here, $E_{y}, E_{z}$, and $H_{x}$ are the corresponding components of the local electric and magnetic fields at the position of particle $i$ ), the interacting functions are

$$
\begin{gathered}
F_{p p}\left(\boldsymbol{r}_{i j}\right)=\hat{z} \cdot \underline{\underline{\Gamma}}^{(p)}\left(\boldsymbol{r}_{i}, \boldsymbol{r}_{j}\right) \cdot \hat{z}, \\
F_{p q}\left(\boldsymbol{r}_{i j}\right)=\hat{z} \cdot \underline{\underline{\underline{\Gamma}}}^{(q)}\left(\boldsymbol{r}_{i}, \boldsymbol{r}_{j}\right) \cdot \hat{y} \hat{z}, \\
F_{p m}\left(\boldsymbol{r}_{i j}\right)=-\hat{z} \cdot \frac{1}{i \omega \epsilon_{0}} \nabla \times\left[\underline{\underline{\Gamma}}^{(m)}\left(\boldsymbol{r}_{i}, \boldsymbol{r}_{j}\right) \cdot \hat{x}\right], \\
F_{q p}\left(\boldsymbol{r}_{i j}\right)=\left(\hat{y} \cdot \partial_{z}+\hat{z} \cdot \partial_{y}\right)\left[\underline{\underline{\Gamma}}^{(p)}\left(\boldsymbol{r}_{i}, \boldsymbol{r}_{j}\right) \cdot \hat{z}\right], \\
F_{q q}\left(\boldsymbol{r}_{i j}\right)=\left(\hat{y} \cdot \partial_{z}+\hat{z} \cdot \partial_{y}\right)\left[\underline{\underline{\underline{\Gamma}}}^{(q)}\left(\boldsymbol{r}_{i}, \boldsymbol{r}_{j}\right) \cdot \hat{y} \hat{z}\right],
\end{gathered}
$$




$$
\begin{gathered}
F_{q m}\left(\boldsymbol{r}_{i j}\right)=-\left(\hat{y} \cdot \partial_{z}+\hat{z} \cdot \partial_{y}\right) \frac{1}{i \omega \epsilon_{0}} \nabla \times\left[\underline{\underline{\Gamma}}^{(m)}\left(\boldsymbol{r}_{i}, \boldsymbol{r}_{j}\right) \cdot \hat{x}\right], \\
F_{m p}\left(\boldsymbol{r}_{i j}\right)=\hat{x} \cdot \frac{1}{i \omega \mu_{0}} \nabla \times\left[\underline{\underline{\Gamma}}^{(p)}\left(\boldsymbol{r}_{i}, \boldsymbol{r}_{j}\right) \cdot \hat{z}\right], \\
F_{m q}\left(\boldsymbol{r}_{i j}\right)=\hat{x} \cdot \frac{1}{i \omega \mu_{0}} \nabla \times\left[\underline{\underline{\Gamma}}^{(q)}\left(\boldsymbol{r}_{i}, \boldsymbol{r}_{j}\right) \cdot \hat{y} \hat{z}\right], \\
F_{m m}\left(\boldsymbol{r}_{i j}\right)=\hat{x} \cdot \underline{\underline{\Gamma}}^{(m)}\left(\boldsymbol{r}_{i}, \boldsymbol{r}_{j}\right) \cdot \hat{x} .
\end{gathered}
$$

Here, $\underline{\underline{\Gamma}}^{(p)}\left(\boldsymbol{r}_{i}, \boldsymbol{r}_{j}\right)$ and $\underset{\underline{\underline{\Gamma}}}{(q)}\left(\boldsymbol{r}_{i}, \boldsymbol{r}_{j}\right)$ are electric-type dyadic Green functions of the electric dipole and electric quadrupole, respectively, while $\underline{\underline{\Gamma}}^{(m)}\left(\boldsymbol{r}_{i}, \boldsymbol{r}_{j}\right)$ is the magnetic-type dyadic Green function of the magnetic dipole. Notice that $\Gamma_{\underline{\equiv}}^{(q)}$ $\times\left(\boldsymbol{r}_{i}, \boldsymbol{r}_{j}\right)$ is a rank-3 dyadic because the electric quadrupole is a rank-2 dyadic. In Eqs. (A1) and (A2), $\boldsymbol{r}_{i}$ and $\boldsymbol{r}_{j}$ are the position vectors of particles $i$ and $j$, respectively, $\boldsymbol{r}_{i j}=\boldsymbol{r}_{i}-\boldsymbol{r}_{j}$. $\underline{\underline{\Gamma}}^{(p)}\left(\boldsymbol{r}_{i}, \boldsymbol{r}_{j}\right), \underline{\underline{\Gamma}}^{(q)}\left(\boldsymbol{r}_{i}, \boldsymbol{r}_{j}\right)$, and $\Gamma^{(m)}\left(\boldsymbol{r}_{i}, \boldsymbol{r}_{j}\right)$ are known functions when the positions of all the particles and the source dipole are given. $\alpha_{p i}, \alpha_{q i}$, and $\alpha_{m i}$ are electric dipole, electric quadrupole, and magnetic dipole polarizabilities, which are determined as

$$
\begin{gathered}
\alpha_{p i}=-\frac{6 \pi i \epsilon_{0} c_{1}^{\mathrm{TM}}}{k_{0}^{3}}, \\
\alpha_{q i}=-\frac{40 \pi i \epsilon_{0} c_{2}^{\mathrm{TM}}}{k_{0}^{5}}, \\
\alpha_{m i}=-\frac{6 \pi i c_{1}^{\mathrm{TE}}}{k_{0}^{3}} .
\end{gathered}
$$

$c_{1}^{\mathrm{TM}}$ and $c_{2}^{\mathrm{TM}}$ are the scattering coefficients of the TM mode of orders 1 and 2 in Mie's scattering theory, as discussed in Sec. II. $c_{1}^{\mathrm{TE}}$ is the scattering coefficient of the TE mode of order 1 , which is determined from $c_{n}^{\mathrm{TE}}=-U_{n}^{\mathrm{TE}} /\left(U_{n}^{\mathrm{TE}}+i V_{n}^{\mathrm{TE}}\right)$, and the formula for $U_{n}^{\mathrm{TE}}$ and $V_{n}^{\mathrm{TE}}$ are analogous to those for $U_{n}^{\mathrm{TM}}$ and $V_{n}^{\mathrm{TM}}$ by substituting $\epsilon$ with $\mu .^{5}$ For a system of $N$ particles, Eq. (A1) provides us with a set of $3 N$ equations. If we use Eq. (A1) instead of Eq. (2), induced electric quadrupoles and magnetic dipoles are included in the system. However, as we discussed in the main text, their influence is indeed very small in all the examples discussed, mainly because the scattering coefficients $c_{2}^{\mathrm{TM}}$ and $c_{1}^{\mathrm{TE}}$ are much smaller than $c_{1}^{\mathrm{TM}}$ when the system is operating near the dipole resonance of the particles. *engheta@ee.upenn.edu

${ }^{1}$ S. A. Maier and H. A. Atwater, J. Appl. Phys. 98, 011101 (2005).

${ }^{2}$ R. D. Averitt, D. Sarkar, and N. J. Halas, Phys. Rev. Lett. 78, 4217 (1997).

${ }^{3}$ S. J. Oldenburg, G. D. Hale, J. B. Jackson, and N. J. Halasa, Appl. Phys. Lett. 75, 1063 (1999).

${ }^{4}$ A. F. Koenderink and A. Polman, Phys. Rev. B 74, 033402 (2006).

${ }^{5}$ A. Alu and N. Engheta, J. Appl. Phys. 97, 094310 (2005).

${ }^{6}$ K. Aslan, I. Gryczynski, J. Malicka, E. Matveeva, J. R. Lakowicz, and C. D. Geddes, Curr. Opin. Biotechnol. 16, 55 (2005).

${ }^{7}$ S. Kuhn, U. Hakanson, L. Rogobete, and V. Sandoghdar, Phys. Rev. Lett. 97, 017402 (2006).

${ }^{8}$ A. L. Mattheyses and D. Axelrod, J. Biomed. Opt. 10, 054007 (2005).

${ }^{9}$ H. Gersen, M. F. Garcia-Parajo, L. Novotny, J. A. Veerman, L. Kuipers, and N. F. van Hulst, Phys. Rev. Lett. 85, 5312 (2000).

${ }^{10}$ K. Sarychev, D. A. Genov, A. Wei, and V. M. Shalaev, Proc. SPIE 5218, 81 (2003).

${ }^{11}$ P. Muhlschlegel, H. J. Eisler, O. J. F. Martin, B. Hecht, and D. W. Pohl, Science 308, 1607 (2005).

${ }^{12} \mathrm{~J}$. Li and N. Engheta, IEEE Trans. Antennas Propag. 55 (11), 3018 (2007).
${ }^{13}$ J. Li, A. Salandrino, and N. Engheta, Proceedings of the International Workshop on Antenna Technology, White Plains, New York, 2006, p. 432.

${ }^{14}$ P. B. Johnson and R. W. Christy, Phys. Rev. B 6, 4370 (1972).

${ }^{15}$ H. Yagi, Proc. IRE 16, 715 (1928).

${ }^{16}$ J. D. Kraus and R. J. Marhefka, Antennas for All Applications, 3rd ed. (McGraw-Hill, New York, 2002).

${ }^{17}$ T. Kalkbrenner, M. Ramstein, J. Mlynek, and V. Sandoghdar, J. Microsc. 202, 72 (2002).

${ }^{18}$ P. Olk, J. Renger, T. Hartling, M. T. Wenzel, and L. M. Eng, Nano Lett. 7, 1736 (2007).

${ }^{19}$ T. Kalkbrenner, U. Hakansan, and V. Sandoghdar, Nano Lett. 4, 2309 (2004).

${ }^{20}$ After we submitted this paper on January 29, 2007, we were informed by H. F. Hofmann about their paper "Design Parameters for a Nano-Antenna Yagi-Uda Antenna" [H. F. Hoffman, T. Kosako, and Y. Kadoya, arXiv:cond-mat/0703595 (unpublished)] posted on March 23, 2007 in http://arxiv.org (later it was published [New J. Phys. 9, 217 (2007)]). In their work, halfwavelength linear optical antenna elements made of plasmonic materials are used as reflectors and directors, which are different from the nanoshell particles we considered here for reflectors and directors. 